\title{
Control of Pipe Cutting Robot: A More Effective Method
}

\author{
Quoc Bao DIEP \\ Faculty of Electrical Engineering and Computer Science, VSB-Technical University of Ostrava, \\ 17. listopadu 15, 70833 Ostrava, Czech Republic \\ diepquocbao@gmail.com \\ (Received: 04-August-2017; accepted: 04-October-2017; published: 30-November-2017) \\ DOI: http://dx.doi.org/10.25073/jaec.201712.82
}

\begin{abstract}
This paper presents a pipe cutting Robot system with two different cutting methods: the method with the end-effector moves on cutting path and direction while the stationary pipe and the method with the end-effector moves on a straight line while the rotating pipe to create the desired cutting path and direction. The cutting trajectory are described, the Robot model is constructed, solving the inverse kinematics, planning the trajectory of motion, simulating and controlling Robot in Matlab, and designing the experimental Robot to verify. The results of the two methods are compared to point out a better one. This research builds up an important foundation for choosing an effective method for pipe cutting Robot in industry.
\end{abstract}

\section{Keywords}

Cutting robot, inverse kinematics, pipe cutting, robot control, trajectory planning.

\section{Introduction}

In industry, gases and liquids are transported daily by pipelines and these pipeline systems paired together in a complex way. To create them, the steel pipes are cut and welded together. However, this is not simple because there are complex joints that require cutting and welding paths to be complicated in which the trajectory and direction change continuously. With that requirement, conventional tools cannot be implemented and the application of robots is necessary.

The use of robot for cutting pipe has become very popular in the world. In Vietnam, this technique has not yet been widely applied, and there are few scientific publications in this field.

In [1, the authors mentioned a Delta Robot for cutting high-speed laser with the numerous advantages of robot: higher stiffness, fewer joints, the ability of transporting heavier loads, and higher accuracy. The main drawback is the small workspace, and this paper also does not mention much about the application of Delta Robot to cut steel pipes.

In [2], the authors presented a pipe cutting technique that included a pipe cutting Robot that the robot arm moves and the pipe is stationary during cutting. The authors successfully builds 3D simulation and experimental model, solving the inverse kinematics, planning the trajectory as well as designs Robot controller. The results of simulated and experimental errors are provided. However, the authors only stopped at the method of the end-effector moves while the stationary pipe without mentioning their coordinated motion.

In this paper, the author presents another method of cutting pipe more effectively with a 6 degrees of freedom pipe cutting Robot, consisting of 5 degrees of freedom robot arm and the 
degree of freedom created by the rotating motion of a pipe. With this method, the end-effector will only move on a straight line, and the pipe will rotate in conjunction with the movement of the end-effector to create cutting path in reality. This result is compared with the results in [2] to point out that this cutting method is better than the cutting method in [2].

\section{Pipe Cutting Problem}

The cutting paths and cutting directions can happen many cases, depending on pipeline assembly position and welding conditions. This paper will focus on Hyperbolic Paraboloid Pringles; a common path is created by two intersecting pipes as shown in Fig. 1.

\subsection{Cutting Path}

In coordinate frame $\{-1\}$, place pipes $R_{1}$ and $R_{2}$ which have center lines coincident with $Z_{-1}$ axis, each pipe equation is given by Eq. (1):

$$
\left\{\begin{array}{l}
x_{n}^{2}+y_{n}^{2}=R_{n}^{2}, \\
-\frac{1}{2} L_{n} \leq z_{n} \leq \frac{1}{2} L_{n},
\end{array}\right.
$$

where

- $L, R$ : the length and radius of two pipes,

- $x, y, z$ : coordinates of two pipes and

- $n=1$ : pipe $R_{1}, n=2$ : pipe $R_{2}$.

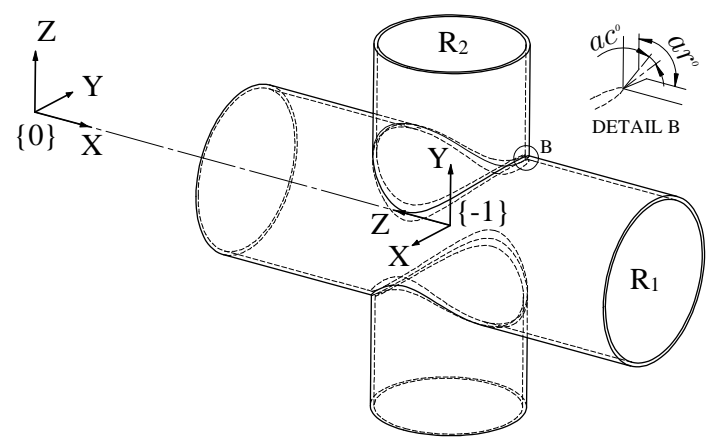

Fig. 1: The intersection of two pipes.
When turning pipe $R_{2}$ an angle $a r^{\circ}$ around $X_{-1}$ axis (for example $90^{\circ}$, see Fig. 1), we have Eq. (2):

$$
\left[\begin{array}{l}
x_{2}^{\prime} \\
y_{2}^{\prime} \\
z_{2}^{\prime}
\end{array}\right]=\left[\begin{array}{ccc}
1 & 0 & 0 \\
0 & c_{a r} & -s_{a r} \\
0 & s_{a r} & c_{a r}
\end{array}\right]\left[\begin{array}{l}
x_{2} \\
y_{2} \\
z_{2}
\end{array}\right],
$$

where

- ar: angle between $R_{1}$ and $R_{2}$ and

- $s_{a r}: \sin a r, c_{a r}: \cos a r$.

Based on Eq. (1) and Eq. (2), we obtain the equation of pipe $R_{2}$ after turning $a r^{\circ}$ as Eq. (3):

$$
\left\{\begin{array}{l}
x_{2}^{2}+\left(y_{2} c_{a r}-z_{2} s_{a r}\right)^{2}=R_{2}^{2}, \\
-\frac{1}{2} L_{2} \leq y_{2} s_{a r}+z_{2} c_{a r} \leq \frac{1}{2} L_{2} .
\end{array}\right.
$$

From Eq. (1), Eq. (2) and Eq. (3), we have the locus of the intersection of two pipes as Eq. (4):

$$
\left\{\begin{array}{l}
x^{2}+y^{2}=R_{1}^{2}, \\
x^{2}+\left(y c_{a r}-z s_{a r}\right)^{2}=R_{2}^{2} .
\end{array}\right.
$$

Assuming that $R_{1} \geq R_{2}$, set:

$$
\left\{\begin{array}{l}
R_{2} s_{\varphi}=x \\
R_{2} c_{\varphi}=y c_{a r}-z s_{a r}
\end{array}\right.
$$

where $0 \leq \varphi \leq 2 \pi, s_{\varphi}: \sin \varphi, c_{\varphi}: \cos \varphi$.

Based on Eq. (4) and Eq. (5), we obtain the locus of the intersection of two pipes in the coordinate frame $\{-1\}$ as Eq. (6), Eq. (7) and Eq. (8):

$$
\begin{aligned}
& x=R_{2} s_{\varphi}, \\
& y= \pm \sqrt{R_{1}^{2}-\left(R_{2} s_{\varphi}\right)^{2}}, \\
& z=\frac{-R_{2} c_{\varphi} \pm c_{a r} \sqrt{R_{1}^{2}-\left(R_{2} s_{\varphi}\right)^{2}}}{s_{a r}} .
\end{aligned}
$$

Set up the coordinate frame $\{0\}$ so that the $X_{0}$ axis is coincided and reversed with the $Z_{-1}$ axis. The distance from $\{-1\}$ to $\{0\}$ is $d_{0}$ (see Fig. 11). The coordinate frame $\{0\}$ is fixed and coordinate frame $\{-1\}$ can rotate around $Z_{0}$ an angle $\theta_{0}$. The locus of the intersection of two 
pipes $R_{1}$ and $R_{2}$ in the coordinate frame $\{0\}$ is given by Eq. (9), Eq. (10), Eq. (11) and (12):

$$
Q_{1}=\left[\begin{array}{cccc}
0 & 0 & -1 & d_{0} \\
-c_{0} & s_{0} & 0 & 0 \\
s_{0} & c_{0} & 0 & 0 \\
0 & 0 & 0 & 1
\end{array}\right]\left[\begin{array}{c}
x \\
y \\
z \\
1
\end{array}\right]=\left[\begin{array}{c}
Q_{1 x} \\
Q_{1 y} \\
Q_{1 z} \\
1
\end{array}\right],
$$

where

$$
\begin{aligned}
& Q_{1 x}=d_{0}-\frac{-R_{2} c_{\varphi} \pm c_{a r} \sqrt{R_{1}^{2}-\left(R_{2} s_{\varphi}\right)^{2}}}{s_{a r}} \\
& Q_{1 y}=-c_{0} R_{2} s_{\varphi} \pm s_{0} \sqrt{R_{1}^{2}-\left(R_{2} s_{\varphi}\right)^{2}} \\
& Q_{1 z}=s_{0} R_{2} s_{\varphi} \pm c_{0} \sqrt{R_{1}^{2}-\left(R_{2} s_{\varphi}\right)^{2}}
\end{aligned}
$$

$s_{0}: \sin \theta_{0}, c_{0}: \cos \theta_{0}$.

When cutting, pipe $R_{2}$ must be re-applied an angle $-a r^{\circ}$ so that the center line of $R_{2}$ coincides with $Z_{-1}$ axis. The cutting path of pipe $R_{2}$ is given by Eq. (13), Eq. (14), Eq. (15) and Eq. 16.

$$
Q_{2}=\left[\begin{array}{ccc}
1 & 0 & -1 \\
0 & c_{-a r} & -s_{-a r} \\
0 & s_{-a r} & c_{-a r}
\end{array}\right]\left[\begin{array}{l}
Q_{1 x} \\
Q_{1 y} \\
Q_{1 z}
\end{array}\right]=\left[\begin{array}{l}
Q_{2 x} \\
Q_{2 y} \\
Q_{2 z}
\end{array}\right],
$$

where

$$
\begin{aligned}
Q_{2 x} & =d_{0}-\frac{-R_{2} c_{\varphi} \pm c_{a r} \sqrt{R_{1}^{2}-\left(R_{2} s_{\varphi}\right)^{2}}}{s_{a r}} \\
Q_{2 y} & =c_{a r}\left(-c_{0} R_{2} s_{\varphi} \pm s_{0} \sqrt{R_{1}^{2}-\left(R_{2} s_{\varphi}\right)^{2}}\right) \\
& +s_{a r}\left(s_{0} R_{2} s_{\varphi} \pm c_{0} \sqrt{R_{1}^{2}-\left(R_{2} s_{\varphi}\right)^{2}}\right), \\
Q_{2 z} & =-s_{a r}\left(-c_{0} R_{2} s_{\varphi} \pm s_{0} \sqrt{R_{1}^{2}-\left(R_{2} s_{\varphi}\right)^{2}}\right) \\
& +c_{a r}\left(s_{0} R_{2} s_{\varphi} \pm c_{0} \sqrt{R_{1}^{2}-\left(R_{2} s_{\varphi}\right)^{2}}\right)
\end{aligned}
$$

Equation (9), Eq. (10), Eq. (11), Eq. (12), Eq. (13), Eq. (14), Eq. (15) and Eq. 16) describe the paths in which the end-effector moves on them in the coordinate frame $\{0\}$ when cutting.

\subsection{Cutting Direction}

Beside cutting path, we must pay attention to the cutting direction. With each cutting point which many directions to go through, but only one direction is reasonable with the requirements about pipe welding conditions [3], depending on the cutting angle [4] and the position of the point, shown in Fig. 2. Cutting direction changes continuously during cutting process.

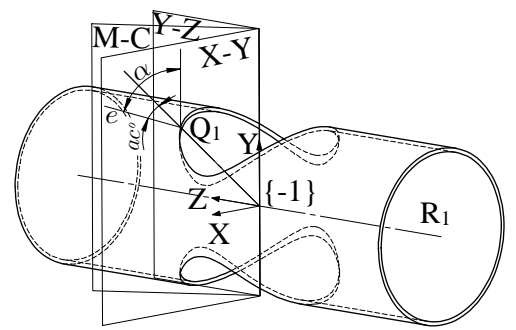

Fig. 2: Cutting direction of pipe $R_{1}$.

In the coordinate frame $\{-1\}$, plane $(M, C)$ contains $Y_{-1}$ axis and passes cutting point $Q_{1}$. In plane $(M, C), e$ is the line that contains cutting direction and passes cutting point $Q_{1}, \alpha$ is the angle between $e$ and $Y_{-1}$ axis (given by Eq. (17) and Eq. (18)). $\beta$ is the angle between plane $(M, C)$ and $(Y, Z)$.

$$
\begin{aligned}
\alpha & =Y_{-1}^{\widehat{O_{-1}}} Q_{1}+a c \\
Y_{-1} \widehat{O_{-1}} Q_{1} & =\arctan 2\left(\sqrt{Q_{1 x}^{2}+Q_{1 z}^{2}}, Q_{1 y}\right) .
\end{aligned}
$$

$a c$ : the standard cutting angle is given before [4] (see Fig. 1] and Fig. 2).

$e$ is found by rotating an imaginary line through $Q_{1}$ and paralleling $Y_{-1}$ axis with an angle $\beta$ around $Y_{-1}$ axis, and then rotating this line with an angle $\alpha$ around $Q_{1}$. e is the line containing the direction we need.

The cutting direction in the coordinate frame $\{-1\}$ is a $3 \times 3$ matrix and given by Eq. (19):

$$
H_{-1}=R_{Y}(\alpha) R_{X}(\beta)=\left[\begin{array}{ccc}
c_{\beta} & s_{\alpha} s_{\beta} & c_{\alpha} s_{\beta} \\
0 & c_{\alpha} & -s_{\alpha} \\
-s_{\beta} & s_{\alpha} c_{\beta} & c_{\alpha} c_{\beta}
\end{array}\right]
$$


From Eq. (19), we obtain the cutting direction in the coordinate frame $\{0\}$ as Eq. 20\}:

$$
\begin{aligned}
& H_{0}={ }_{0}^{-1} R H_{-1} \\
& =\left[\begin{array}{ccc}
s_{\beta} & -s_{\alpha} c_{\beta} & -c_{\alpha} c_{\beta} \\
-c_{0} c_{\beta} & -c_{0} s_{\alpha} s_{\beta}+s_{0} c_{\alpha} & -c_{0} c_{\alpha} s_{\beta}-s_{0} s_{\alpha} \\
s_{0} c_{\beta} & s_{0} s_{\alpha} s_{\beta}+c_{0} c_{\alpha} & s_{0} c_{\alpha} s_{\beta}-c_{0} s_{\alpha}
\end{array}\right]
\end{aligned}
$$

\subsection{Cutting Method}

There are two general cases of cutting: static pipe while moving end-effector 2 and rotating pipe while moving end-effector. Especially, the end-effector moves on a straight section with cutting direction following to Eq. (20). The combination of rotating pipe while moving end-effector on a straight section is given by Eq. (21) and Eq. 22). It will create Hyperbolic Paraboloid Pringles like the reality. This case is described in Fig. 3.

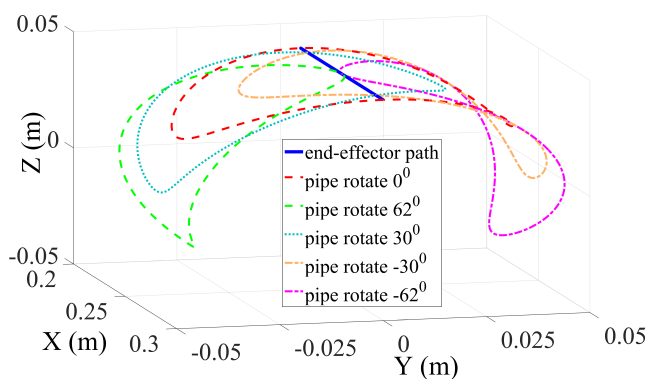

Fig. 3: The combination of rotating pipe while moving end-effector on a straight section.

Trajectory of end-effector is a parallel straight section with $X_{0}$ axis, and is located right at the top of the pipe. The straight section belongs to plane $\left(X_{0}, Z_{0}\right)$, in which $Y_{0}=0$ and $Z_{0}=R_{1}$. From Eq. (9), Eq. (10), Eq. (11) and Eq. (12), we infer:

$$
\begin{aligned}
{ }_{-1}^{0} Q_{1} & =\left[\begin{array}{c}
d_{0}-\frac{-R_{2} c_{\varphi} \pm c_{a r} \sqrt{R_{1}^{2}-\left(R_{2} s_{\varphi}\right)^{2}}}{s_{a r}} \\
-c_{0} R_{2} s_{\varphi} \pm s_{0} \sqrt{R_{1}^{2}-\left(R_{2} s_{\varphi}\right)^{2}} \\
s_{0} R_{2} s_{\varphi} \pm c_{0} \sqrt{R_{1}^{2}-\left(R_{2} s_{\varphi}\right)^{2}}
\end{array}\right] \\
= & {\left[\begin{array}{c}
d_{0}-\frac{-R_{2} c_{\varphi} \pm c_{a r} \sqrt{R_{1}^{2}-\left(R_{2} s_{\varphi}\right)^{2}}}{s_{a r}} \\
0 \\
R_{1}
\end{array}\right] . }
\end{aligned}
$$

From Eq. (21), we obtain:

$$
\theta_{0}=\arctan 2\left(\mp R_{2} c_{\varphi}, \sqrt{R_{1}^{2}-\left(R_{2} s_{\varphi}\right)^{2}}\right) .
$$

While the end-effector moves on a straight line in Eq. (21) with direction in Eq. (20), the pipe rotate an angle $\theta_{0}$ in Eq. 22). This combination of motion will create the Hyperbolic Paraboloid Pringles in real.

\section{Robot Model}

The Robot model used in this paper includes 5 degrees of freedom robot arm and a degree of freedom created by the rotating motion of the pipe. This model is shown in Fig. 4.

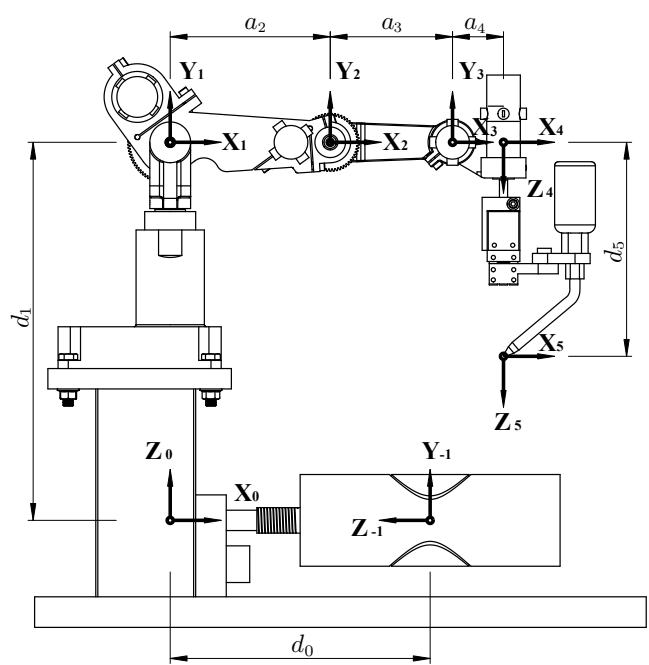

Fig. 4: The model of Robot.

\subsection{Forward Kinematics}

The coordinate frame $\{0\}$ is the global coordinate frame of Robot system. Robot system is divided into two parts in which they combination movements together: a part includes 5 degrees of freedom robot arm and a part created by the rotating motion of a pipe. Parameters of the model are given in Tab. 1 .

Based on the Denavit-Hartenberg convention, we find these transformation matrices 
Tab. 1: Denavit-Hartenberg parameters of Robot.

\begin{tabular}{cccccc}
\hline$i$ & $\begin{array}{c}\alpha_{i} \\
\mathrm{deg}\end{array}$ & $\begin{array}{c}a_{i} \\
\mathrm{~mm}\end{array}$ & $\begin{array}{c}d_{i} \\
\mathrm{~mm}\end{array}$ & $\begin{array}{c}\theta_{i} \\
\mathrm{deg}\end{array}$ & $\begin{array}{c}\text { Operating range } \\
\text { deg }\end{array}$ \\
\hline 0 & 90 & 0 & 260 & $\theta_{0}$ & -180 to 180 \\
1 & 90 & 0 & 371 & $\theta_{1}$ & -140 to 140 \\
2 & 0 & 157 & 0 & $\theta_{2}$ & -45 to 45 \\
3 & 0 & 120 & 0 & $\theta_{3}$ & -90 to 90 \\
4 & 90 & 50 & 0 & $\theta_{4}$ & -145 to 90 \\
5 & 0 & 0 & 210 & $\theta_{5}$ & -180 to 180 \\
\hline
\end{tabular}

${ }_{-1}^{0} T,{ }_{1}^{0} T,{ }_{2}^{1} T,{ }_{3}^{2} T,{ }_{4}^{3} T$ and ${ }_{5}^{4} T$. The transformation matrix ${ }_{-1}^{0} T$ describe position of the pipe in global coordinate frame $\{0\}$, given by Eq. (23):

$$
{ }_{-1}^{0} T=\left[\begin{array}{cccc}
0 & 0 & -1 & d_{0} \\
-c_{0} & s_{0} & 0 & 0 \\
s_{0} & c_{0} & 0 & 0 \\
0 & 0 & 0 & 1
\end{array}\right]
$$

Matrix ${ }_{5}^{0} T$ describes the end-effector direction and position in the coordinate frame $\{0\}$ (see [2] or [5]) is given by Eq. (24):

$$
{ }_{5}^{0} T={ }_{1}^{0} T{ }_{2}^{1} T{ }_{3}^{2} T{ }_{4}^{3} T{ }_{5}^{4} T=\left[\begin{array}{cccc}
n_{x} & a_{x} & o_{x} & p_{x} \\
n_{y} & a_{y} & o_{y} & p_{y} \\
n_{z} & a_{z} & o_{z} & p_{z} \\
0 & 0 & 0 & 1
\end{array}\right],
$$

where

$$
\begin{aligned}
n_{x} & =c_{1} c_{234} c_{5}+s_{1} s_{5}, \\
n_{y} & =s_{1} c_{234} c_{5}-c_{1} s_{5}, \\
n_{z} & =s_{234} c_{5}, \\
a_{x} & =-c_{1} c_{234} s_{5}+s_{1} c_{5}, \\
a_{y} & =-s_{1} c_{234} s_{5}-c_{1} c_{5}, \\
a_{z} & =-s_{234} s_{5}, \\
o_{x} & =c_{1} s_{234} \\
o_{y} & =s_{1} s_{234}, \\
o_{z} & =-c_{234}, \\
p_{x} & =d_{5} c_{1} s_{234}+a_{4} c_{1} c_{234}+a_{3} c_{1} c_{23}+a_{2} c_{1} c_{2}, \\
p_{y} & =d_{5} s_{1} s_{234}+a_{4} s_{1} c_{234}+a_{3} s_{1} c_{23}+a_{2} s_{1} c_{2}, \\
p_{z} & =-d_{5} c_{234}+a_{4} s_{234}+a_{3} s_{23}+a_{2} s_{2}+d_{1},
\end{aligned}
$$

$s_{i}: \sin \theta_{i}, c_{i}: \cos \theta_{i}$,

$s_{i \ldots k}: \sin \left(\theta_{i}+\cdots+\theta_{k}\right), c_{i \ldots k}: \cos \left(\theta_{i}+\cdots+\theta_{k}\right)$.

\subsection{Inverse Kinematics}

Inverse kinematics results in exact positions of joints when position and direction of end-effector is known.

Equating entries $(1,4)$ and $(2,4)$ in the matrix (Eq. (24)), we have $\tan \theta_{1}$ ([2] or [6]) as Eq. (37):

$$
\frac{p_{y}}{p_{x}}=\tan \theta_{1} .
$$

We obtain the first angle as Eq. (38).

$$
\theta_{1}=\arctan 2\left(p_{y}, p_{x}\right) .
$$

By multiplying $s_{1}$ and $c_{1}$ with elements $(1,1)$, $(2,1),(1,2)$ and $(2,2)$ in Eq. (24) then shorten them, we find $\tan \theta_{5}$ as $\mathrm{Eq}(39)$ :

$$
\frac{s_{1} n_{x}-c_{1} n_{y}}{s_{1} o_{x}-c_{1} o_{y}}=\tan \theta_{5} .
$$

Infer $\theta_{5}$ as $\mathrm{Eq}(40)$ :

$$
\theta_{5}=\arctan 2\left(s_{1} n_{x}-c_{1} n_{y}, s_{1} o_{x}-c_{1} o_{y}\right) \text {. }
$$

To find $\theta_{3}$, we need to find $\theta_{234}=\theta_{2}+\theta_{3}+$ $\theta_{4}$. Equating entries $(1,3),(2,3)$ and $(3,3)$ in the matrix Eq. 24), we obtain Eq 41):

$$
\frac{c_{1} a_{x}+s_{1} a_{y}}{-a_{z}}=\tan \theta_{234} .
$$

Infer $\theta_{234}$ as Eq (42):

$$
\theta_{234}=\arctan 2\left(c_{1} a_{x}+s_{1} a_{y},-a_{z}\right) .
$$

After we have $\theta_{1}$ and $\theta_{5}$, we calculate the value of the transformation matrix ${ }_{1}^{0} T$ and ${ }_{5}^{4} T$. Based on the value of the transformation matrix ${ }_{1}^{0} T$, we find ${ }_{4}^{1} T$ as Eq. (43):

$$
\begin{aligned}
{ }_{4}^{1} T & ={ }_{1}^{0} T^{-1}{ }_{5}^{0} T_{5}^{4} T^{-1} \\
& =\left[\begin{array}{cccc}
c_{234} & 0 & s_{234} & a_{4} c_{234}+a_{3} c_{23}+a_{2} c_{2} \\
s_{234} & 0 & -c_{234} & a_{4} s_{234}+a_{3} s_{23}+a_{2} s_{2} \\
0 & 1 & 0 & 0 \\
0 & 0 & 0 & 1
\end{array}\right] .
\end{aligned}
$$

We set:

$$
\left\{\begin{array}{l}
x_{41}=a_{4} c_{234}+a_{3} c_{23}+a_{2} c_{2}, \\
y_{41}=a_{4} s_{234}+a_{3} s_{23}+a_{2} s_{2} .
\end{array}\right.
$$


Rearranging the two equations in Eq. (44), squaring them and then adding the squares gives Eq. (45) and Eq. (46):

$c_{3}=\frac{\left(x_{41}-a_{4} c_{234}\right)^{2}+\left(y_{41}-a_{4} s_{234}\right)^{2}-a_{3}^{2}-a_{2}^{2}}{2 a_{3} a_{2}}$,

$s_{3}= \pm \sqrt{1-c_{3}^{2}}$.

Infer $\theta_{3}$ as Eq 477:

$$
\theta_{3}=\arctan 2\left(s_{3}, c_{3}\right) \text {. }
$$

Set:

$$
\left\{\begin{array}{l}
k_{1}=a_{3} c_{3}+a_{2}, \\
k_{2}=a_{3} s_{3} .
\end{array}\right.
$$

From Eq. (44) and Eq. (48), we obtain:

$$
\left\{\begin{array}{l}
x_{41}-a_{4} c_{234}=k_{1} c_{2}-k_{2} s_{2}, \\
y_{41}-a_{4} s_{234}=k_{1} s_{2}+k_{2} c_{2} .
\end{array}\right.
$$

Set:

$$
\left\{\begin{array}{l}
k_{3}=\sqrt{k_{1}^{2}+k_{2}^{2}}, \\
\gamma=\arctan 2\left(k_{2}, k_{1}\right) .
\end{array}\right.
$$

Based on Eq. 49 and Eq. (50), we obtain Eq. 51).

$$
\left\{\begin{array}{l}
\cos \left(\gamma+\theta_{2}\right)=\frac{x_{41}-a_{4} c_{234}}{k_{3}}, \\
\sin \left(\gamma+\theta_{2}\right)=\frac{y_{41}-a_{4} s_{234}}{k_{3}} .
\end{array}\right.
$$

From Eq. (51), we obtain $\theta_{2}$ ([2 or [7) as Eq. (52):

$$
\begin{aligned}
\theta_{2} & =\arctan 2\left(y_{41}-a_{4} s_{234}, x_{41}-a_{4} c_{234}\right) \\
& -\arctan 2\left(k_{2}, k_{1}\right) .
\end{aligned}
$$

And we have $\theta_{4}$ as Eq. (53):

$$
\theta_{4}=\theta_{234}-\theta_{3}-\theta_{2} \text {. }
$$

\subsection{Trajectory Planning}

Links will be moved concurrently and corporately so that end-effector will follow cutting equation in defined duration $t$.
In Cartesian space, cutting path will be divided into set of points in which the space between these points is very small and equal $\Delta p$. Inverse kinematics is used to define joint variables in joint space corresponding to set of points in Cartesian space ([8], [9] or [10]).

Joint path planning must ensure the continuity of position, velocity, acceleration and cubic polynomial is a suitable choice.

Suppose that $n^{\text {th }}$ joint rotates angles $\theta_{i}$ and $\theta_{i+1}$ in durations $t_{k}$ and $t_{k+1}$ respectively, the cubic polynomial of the form of $n^{\text {th }}$ joint:

$$
\theta_{i}(t)=a_{i 3} t^{3}+a_{i 2} t^{2}+a_{i 1} t+a_{i 0} .
$$

Velocity:

$$
\dot{\theta}_{i}(t)=3 a_{i 3} t^{2}+2 a_{i 2} t+a_{i 1} .
$$

Parameters of the cubic polynomial must be defined based on constraints so that joint path satisfy the continuity of position, velocity and acceleration.

Position constraints:

$$
\begin{aligned}
& \theta_{i}(0)=\theta_{k-1}, \\
& \theta_{i}\left(t_{k}\right)=\theta_{i+1}(0)=\theta_{k}, \\
& \theta_{i+1}\left(t_{k+1}\right)=\theta_{k+1} .
\end{aligned}
$$

Velocity constraints:

$$
\begin{aligned}
& \dot{\theta}_{i}(0)=\dot{\theta}_{k-1}, \\
& \dot{\theta}_{i}\left(t_{k}\right)=\dot{\theta}_{i+1}(0)=\dot{\theta}_{k}, \\
& \dot{\theta}_{i+1}\left(t_{k+1}\right)=\dot{\theta}_{k+1} .
\end{aligned}
$$

Acceleration constraints:

$$
\ddot{\theta}_{i}\left(t_{k}\right)=\ddot{\theta}_{i+1}(0)=\ddot{\theta}_{k} .
$$

The durations are equal: $t_{k}=t_{k+1}=\Delta t$, solving Eq. (56), Eq. (57), Eq. (58), Eq. (59), Eq. 60), Eq. (61) and Eq. 62), we obtain:

$$
\begin{aligned}
a_{i 3} & =\frac{-2\left(\theta_{k}-\theta_{k-1}\right)+\left(\dot{\theta}_{k}+\dot{\theta}_{k-1}\right) \Delta t}{\Delta t^{3}} \\
a_{i 2} & =\frac{6\left(\theta_{k}-\theta_{k-1}\right)-2\left(\dot{\theta}_{k}+2 \dot{\theta}_{k-1}\right) \Delta t}{2 \Delta t^{2}}, \\
a_{i 1} & =\dot{\theta}_{k-1}, \\
a_{i 0} & =\theta_{k-1} .
\end{aligned}
$$


Plugging Eq. (63), Eq. (64), Eq. 65) and (66) into Eq. (54) and Eq. (55), we find that:

$$
\begin{aligned}
\theta_{i}(t) & =\frac{-2\left(\theta_{k}-\theta_{k-1}\right)+\left(\dot{\theta}_{k}+\dot{\theta}_{k-1}\right) \Delta t}{\Delta t^{3}} t^{3} \\
& +\frac{6\left(\theta_{k}-\theta_{k-1}\right)-2\left(\dot{\theta}_{k}+2 \dot{\theta}_{k-1}\right) \Delta t}{2 \Delta t^{2}} t^{2} \\
& +\dot{\theta}_{k-1} t+\theta_{k-1}, \\
\dot{\theta}_{i}(t) & =3 \frac{-2\left(\theta_{k}-\theta_{k-1}\right)+\left(\dot{\theta}_{k}+\dot{\theta}_{k-1}\right) \Delta t}{\Delta t^{3}} t^{2} \\
& +2 \frac{6\left(\theta_{k}-\theta_{k-1}\right)-2\left(\dot{\theta}_{k}+2 \dot{\theta}_{k-1}\right) \Delta t}{2 \Delta t^{2}} t \\
& +\dot{\theta}_{k-1} .
\end{aligned}
$$

\section{Simulation and Control}

Trajectory and direction were planned in Cartesian space. They will be transformed into the joint space. The robot is controlled in the joint space so that the end-effector follows the trajectory and the expected direction. Control flow chart algorithm is given by Fig. 5 .

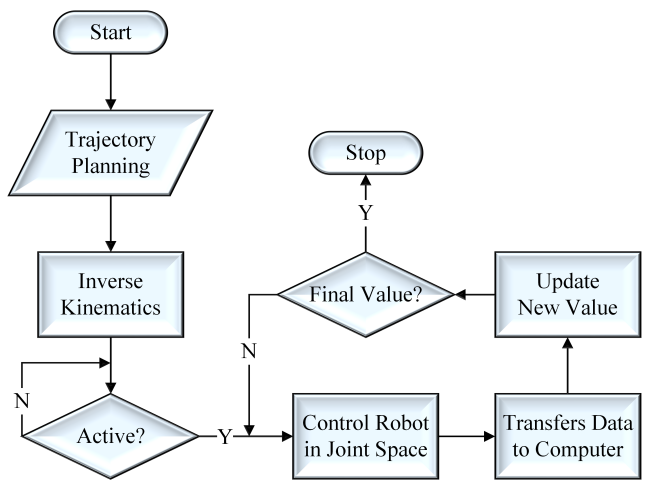

Fig. 5: Control flowchart algorithm.

The robot is drawn by SolidWorks as Fig. 6 . This Robot system is imported into Matlab Simulink as Fig. 7 .

PID transfer function of the first order filter ([11] and [12]) is given by Eq. (69):

$\frac{V(s)}{E(s)}=\frac{K_{d} s+K_{p}+\frac{K_{i}}{s}}{\tau_{f} s+1}=\frac{\frac{K_{d}}{\tau_{f}} s^{2}+\frac{K_{p}}{\tau_{f}} s+\frac{K_{i}}{\tau_{f}}}{s^{2}+\frac{s}{\tau_{f}}}$,

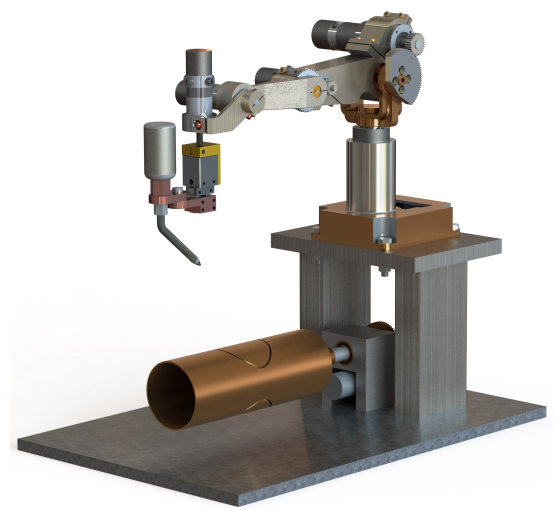

Fig. 6: The 3D system of Robot.

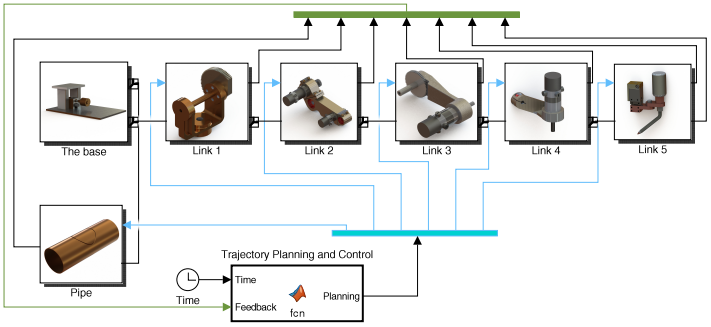

Fig. 7: The Robot system in Matlab Simulink.

where

- $V$ : The output of the controller,

- E: Error between the input value and the feedback value,

- $K_{p}, K_{d}, K_{i}$ : Proportional gain, derivative gain, integral gain and

- $\tau_{f}$ : The time constant of the first order filter.

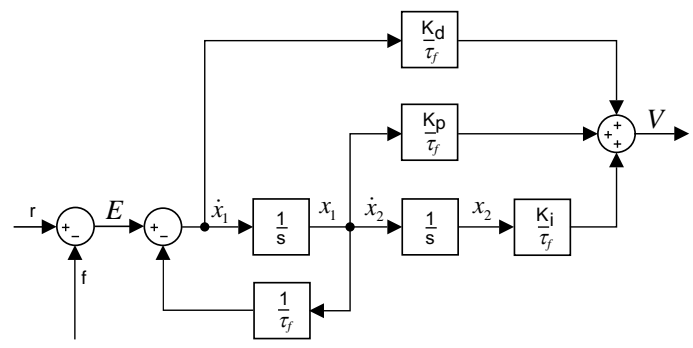

Fig. 8: PID controller with first order filter. 
From Fig. 8, we have:

$$
\begin{aligned}
\dot{x}_{1} & =-\frac{1}{\tau_{f}} x_{1}+e, \\
\dot{x}_{2} & =x_{1}, \\
V & =\frac{K_{i}}{\tau_{f}} x_{2}+\frac{K_{p}}{\tau_{f}} x_{1}+\frac{K_{d}}{\tau_{f}} \dot{x}_{1} \\
& =\left(\frac{K_{p}}{\tau_{f}}-\frac{K_{d}}{\tau_{f}^{2}}\right) x_{1}+\frac{K_{i}}{\tau_{f}} x_{2}+\frac{K_{d}}{\tau_{f}} e .
\end{aligned}
$$

In state space, Eq. (70), Eq. (71) and Eq. (72) are given by Eq. (73) and Eq. (74):

$$
\begin{aligned}
{\left[\begin{array}{c}
\dot{x}_{1} \\
\dot{x}_{2}
\end{array}\right] } & =\left[\begin{array}{cc}
-\frac{1}{\tau_{f}} & 0 \\
1 & 0
\end{array}\right]\left[\begin{array}{l}
x_{1} \\
x_{2}
\end{array}\right]+\left[\begin{array}{l}
1 \\
0
\end{array}\right] e, \\
V & =\left[\frac{K_{p}}{\tau_{f}}-\frac{K_{d}}{\tau_{f}^{2}} \frac{K_{i}}{\tau_{f}}\right]\left[\begin{array}{l}
x_{1} \\
x_{2}
\end{array}\right]+\frac{K_{d}}{\tau_{f}} e .
\end{aligned}
$$

Set:

$$
\begin{aligned}
X & =\left[\begin{array}{l}
x_{1} \\
x_{2}
\end{array}\right] ; \quad \dot{X}=\left[\begin{array}{l}
\dot{x}_{1} \\
\dot{x}_{2}
\end{array}\right], \\
A & =\left[\begin{array}{cc}
-\frac{1}{\tau_{f}} & 0 \\
1 & 0
\end{array}\right] ; \quad B=\left[\begin{array}{l}
1 \\
0
\end{array}\right], \\
C & =\left[\begin{array}{ll}
\frac{K_{p}}{\tau_{f}}-\frac{K_{d}}{\tau_{f}^{2}} & \frac{K_{i}}{\tau_{f}}
\end{array}\right] ; \quad D=\frac{K_{d}}{\tau_{f}} .
\end{aligned}
$$

From Eq. (75), Eq. (76) and Eq. (77), we find that:

$$
\begin{aligned}
& \dot{X}=A X+B e, \\
& V=C X+D e .
\end{aligned}
$$

\section{The simulated results:}

The simulation time is the $30 \mathrm{~s}$. Figure. 9 and Fig. 10 give trajectory and response of six joints in the joint space.

Figure 11 and Fig. 12 are the error graphs of six joints in the joint space. In Fig. 11. Since the static pipe leads to the joint 0 is motionless and errorless, the maximum error belongs to joints 3,4 and 5 with a maximum value of $0.04^{\circ}$. The best activity is joint 1 with a maximum error of $0.0075^{\circ}$. In Fig. 12. Since the end-effector moves on the straight section, joint 1 stays still.

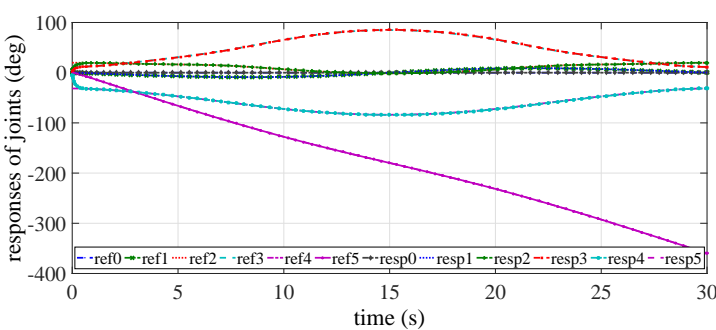

Fig. 9: Trajectory and response of six joints in the case of static pipe.

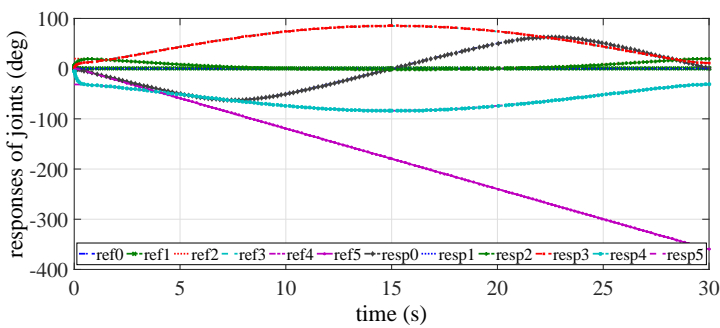

Fig. 10: Trajectory and response of six joints in the case of rotary pipe.

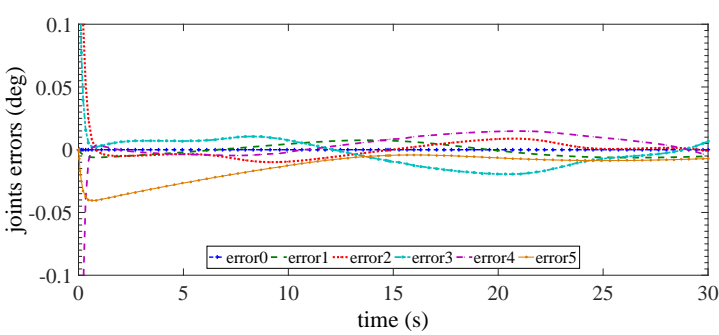

Fig. 11: Joint errors of six joints in the case of static pipe.

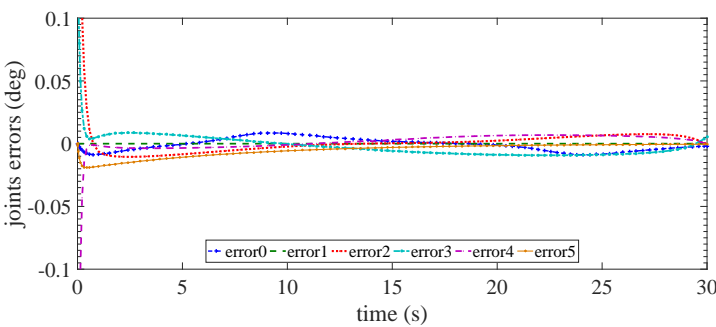

Fig. 12: Joint errors of six joints in the case of rotary pipe.

The maximum error belongs to joint 3 with a value of $0.009^{\circ}$. The best activity is joint 4 with a maximum error of $0.0006^{\circ}$.

Figure 13 and Fig. 14 are the error graphs of the end-effector in the three axes of $X-Y-Z$ in the Cartesian space corresponding to two cases: standing and rotating. In Fig. 13. Position error 


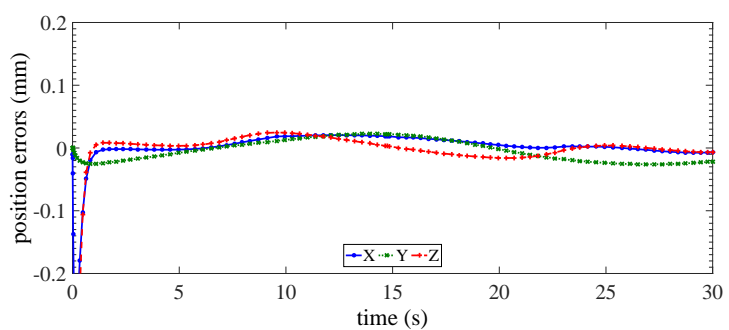

Fig. 13: Position errors of the end-effector in the case of static pipe.

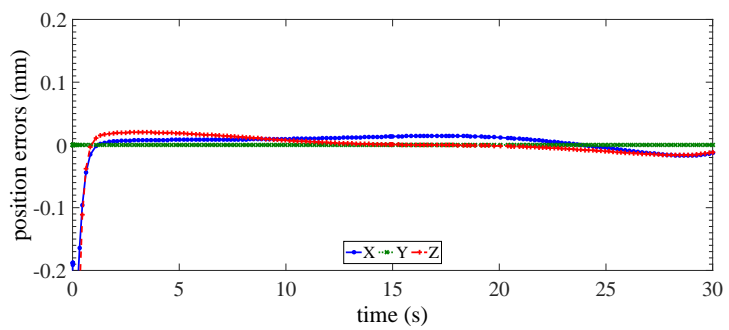

Fig. 14: Position errors of the end-effector in the case of rotary pipe.

ranges from -0.022 to $0.026 \mathrm{~mm}$. In Fig. 14 Position error ranges from -0.021 to $0.020 \mathrm{~mm}$.

Discussion: The simulated results showed that the case of a static pipe cutting was not as good as the case of a rotary pipe cutting.

\section{Experiment}

Figure 15 is the real robot system. Time to finishing work of Robot is set to $30 \mathrm{~s}$. Two microcontrollers will control five harmonic driver motors corresponding to five joints of Robot and a rotary motor. Data obtained from Robot will be transmitted to the computer.

\section{The experimental results:}

Figure 16 and Fig. 17 are the error graphs of six joints in the joint space. In Fig. 16. The maximum error belongs to joints 3 and 4 ; the error ranges from $-0.072^{\circ}$ to $0.079^{\circ}$. In Fig. 17 . The maximum error belongs to joints 3 and 4; the error ranges from $-0.058^{\circ}$ to $0.045^{\circ}$. These errors are smaller in Fig. 16.

Figure 18 and Fig. 19 are the error graphs of the end-effector in the three axes of $X-Y-Z$ in the Cartesian space corresponding to two cases:

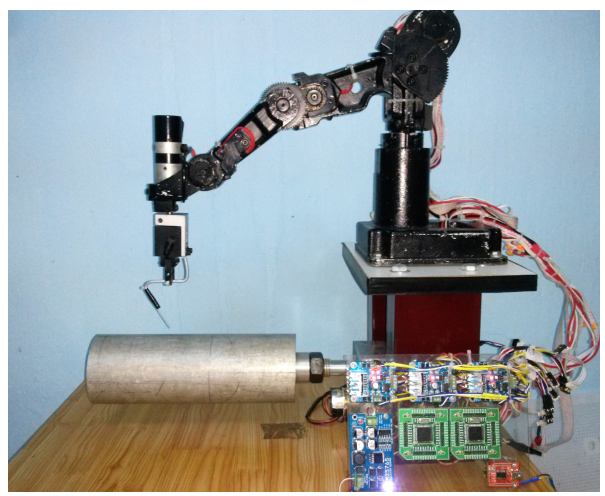

Fig. 15: Experimental Robot system.

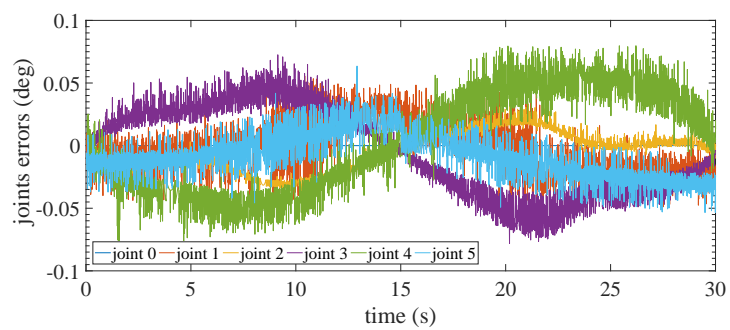

Fig. 16: Joint errors of six joints in the case of static pipe.

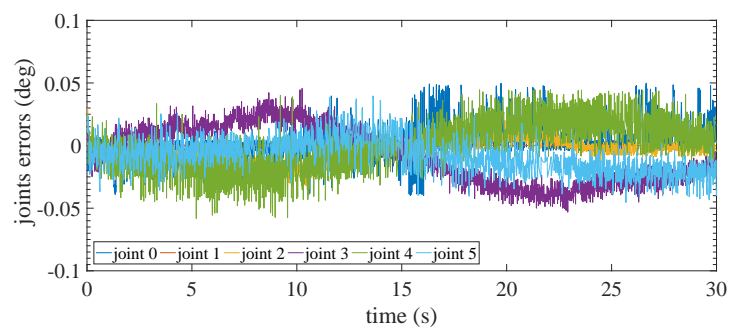

Fig. 17: Joint errors of six joints in the case of rotary pipe.

standing and rotating. We see that the errors in the two graphs range from -0.2 to $0.2 \mathrm{~mm}$. Fig. 18 has a larger error graph and is more oscillating than Fig. 19.

Discussion: The experimental results showed that the case of a rotary pipe cutting was better than the case of a static pipe cutting, with less error and less oscillation error.

\section{Conclusion}

The paper has solved the whole problem: building the cutting trajectory, solving the inverse 


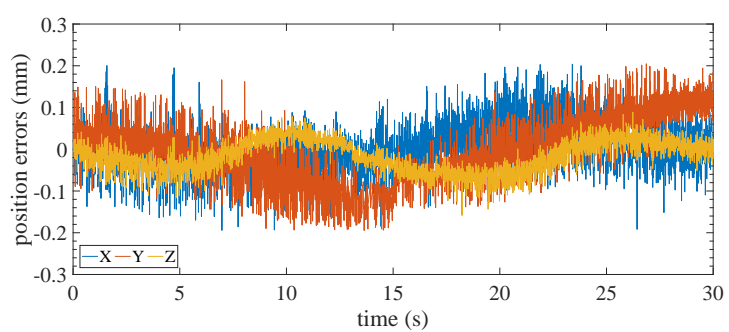

Fig. 18: Position errors of the end-effector in the case of static pipe.

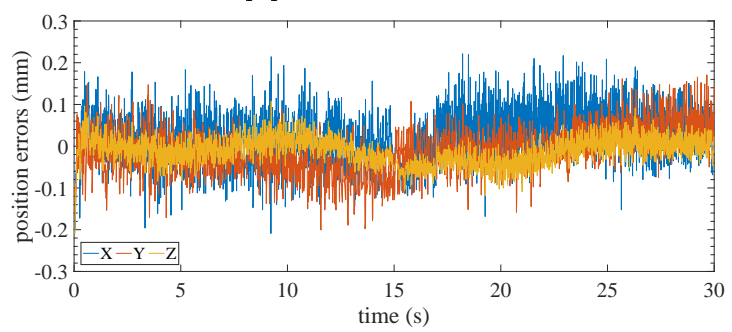

Fig. 19: Position errors of the end-effector in the case of rotary pipe.

kinematics, planning the trajectory of motion, simulating and controlling Robot in reality. More importantly, this paper has developed two different pipe cutting solutions, and gives the comparative results between the two ones in both simulation and experiment. These comparative results show that method of the endeffector moves on a straight line while the rotating pipe to create the cutting path and direction for better than method of the end-effector moves on cutting path and direction while the stationary pipe. This conclusion is an important note that we should design the robot arm and the pipe coordinate movement together, bring the best effect.

\section{Acknowledgment}

The work was supported by Professor Ivan Zelinka, Ton Duc Thang University and Professor Nguyen Tan Tien, "Hi-Tech Mechatronics Laboratory" Ho Chi Minh City University of Technology.

\section{References}

[1] MOHARANA, B., R. GUPTA and B. K. KUSHWAHA. Optimization and Design of a Laser-Cutting Machine using Delta Robot. International Journal of Engineering Trends and Technology. 2014, vol. 10, no. 4, pp. 176-179.

[2] QUOC BAO, D., T. T. PHAN and T. T. NGUYEN. Study on Control of Pipe Cutting Robot. In: International Symposium on Mechatronics and Robotics. Ho Chi Minh City: HCMUT, 2013, pp. 118-123.

[3] Chapter 2. MCALLISTER, E. W. Pipeline Rules of Thumb Handbook. 8th Edition. Boston: Elsevier/Gulf Professional Publishing, 2014, pp. 64-91. .

[4] Chapter 17. MENON, E. S. Pipeline Planning and Construction Field Manual. Waltham: Gulf Professional Publishing, 2011, pp. 357-378.

[5] VERMA, A. and V. A. DESHPANDE. End-effector position analysis of SCORBOT-ER-Vplus Robot. International Journal of Smart Home. 2011, vol. 5, no. 1 , pp. 1-6.

[6] DESHPANDE, V. A. and P. M. GEORGE. Analytical solution for inverse kinematics of SCORBOT-ER-Vplus Robot. International Journal of Emerging Technology and Advanced Engineering. 2012, vol. 2, iss. 3, pp. $478-481$.

[7] XU, D., C. A. ACOSTA CALDERON, J. Q. GAN, H. HU and M. TAN. An analysis of the inverse kinematics for a 5DOF manipulator. International Journal of Automation and Computing. 2005, vol. 2, iss. 2, pp. 114-124.

[8] JAZAR, R. N. Theory of Applied Robotics: Kinematics, Dynamics, and Control. 2nd Edition. New York: Springer, 2010.

[9] CRAIG, J. J. Introduction to Robotics: $M e-$ chanics and Control. 3rd Edition. Upper Saddle River: Pearson/Prentice Hall, 2005. 
[10] TONDU, B. and S. A. BAZAZ. The ThreeCubic Method: An Optimal Online Robot Joint Trajectory Generator under Velocity, Acceleration, and Wandering Constraints. The International Journal of Robotics Research. 1999, vol. 18, iss. 9, pp. 893-901.

[11] SKOGESTAD, S. Simple Analytic Rules for Model Reduction and PID Controller Tuning. Journal of Process Control. 2003, vol. 13, iss. 4, pp. 291-309.

[12] AGRAWAL, S., V. KUMAR, K. P. S. RANA and P. MISHRA. Optimization of PID controller with first order noise filter. In: International Conference on Futuristic Trends on Computational Analysis and Knowledge Management. Noida: IEEE, 2015, pp. 226-231.

\section{About Authors}

Quoc Bao DIEP received the M.Sc. degree from Ho Chi Minh City University of Technology. He is a Ph.D. student at the Faculty of Electrical Engineering and Computer Science, VSB-Technical University of Ostrava. His research interests include industrial robot, 3D simulation, and intelligent control.

"This is an Open Access article distributed under the terms of the Creative Commons Attribution License, which permits unrestricted use, distribution, and reproduction in any medium, provided the original work is properly cited (CC BY 4.0)." 